\title{
ABORDAGENS NA PESQUISA EM EDUCAÇÃO MATEMÁTICA: ALGUMAS REFLEXÕES E PERSPECTIVAS EPISTEMOLÓGICAS
}

\section{APPROACHES IN RESEARCH IN MATHEMATICAL EDUCATION: SOME REFLECTIONS AND EPISTEMOLOGICAL PERSPECTIVES}

\section{ENFOQUES EN LA INVESTIGACIÓN EN EDUCACIÓN MATEMÁTICA: ALGUNAS REFLEXIONES Y PERSPECTIVAS EPISTEMOLÓGICAS}

\author{
José Ricardo e Souza Mafra ${ }^{1}$ \\ Pedro Franco de Sá2
}

\begin{abstract}
Resumo: Este trabalho apresenta um estudo, objetivando realizar uma reflexão sobre abordagens de pesquisa em Educação Matemática, no Brasil e a nível mundial. Apresenta elementos referenciais para se pensar e refletir sobre a atividade de pesquisa educacional, relativa à educação matemática, através de diversas fontes bibliográficas, tais como: livros, periódicos, anais de eventos de educação matemática ou educação, relacionadas ao tema da pesquisa. Os resultados obtidos indicam que a diversidade de abordagens de pesquisa da Educação Matemática advém das diversas correntes metodológicas, configuradas nas últimas décadas, com forte ênfase na pesquisa em Ciências Sociais e na Educação, projetando uma espécie de espalhamento epistemológico. Estas projeções epistemológicas, apontam desafios atuais, tais como: produzir resultados generalizáveis e que se aproximem da Educação Básica; desenvolver repositórios das tendências de pesquisa; aumentar a quantidade de pesquisas em rede e a produção de materiais adequados para quem não gosta de matemática.
\end{abstract}

Palavras-chave: Educação Matemática, Abordagens metodológicas, Epistemologia da Educação Matemática.

\begin{abstract}
This work presents a study, aiming to reflect on research approaches in Mathematics Education, in Brazil and worldwide. It presents reference elements to think about and reflect on the educational research activity, related to mathematics education, through several bibliographic sources, such as: books, periodicals, annals of mathematics education or education events, related to the research theme. The results obtained indicate that the diversity of research approaches in Mathematical Education comes from the different methodological currents, configured in the last decades, with a strong emphasis on research in Social Sciences and Education, projecting a kind of
\end{abstract}

\footnotetext{
${ }^{1}$ Universidade Federal do Oeste do Pará. Santarém, Pará, Brasil.

${ }^{2}$ Universidade do Estado do Pará, Belém, Pará, Brasil.
} 
epistemological spread. These epistemological projections, point to current challenges, such as: producing generalizable results and approaching Basic Education; develop research trend repositories; increase the amount of network research and the production of suitable materials for those who do not like mathematics.

Keywords: Mathematics Education, Methodological Approaches, Epistemology of Mathematics Education.

Resumen: Este trabajo presenta un estúdio, con el objetivo de llevar a cabo una reflexión sobre los enfoques de investigación en Educación Matemática, en Brasil y en todo el mundo. Presenta elementos de referencia para pensar y reflexionar sobre la actividad de investigación educativa, relacionada con la educación matemática, a través de varias fuentes bibliográficas, tales como: libros, publicaciones periódicas, anales de educación matemática o eventos educativos, relacionados con el tema de investigación. Los resultados obtenidos indican que la diversidad de enfoques de investigación en Educación Matemática proviene de las diferentes corrientes metodológicas, configuradas en las últimas décadas, con un fuerte énfasis en la investigación en Ciencias Sociales y Educación, proyectando una especie de diseminación epistemológica. Estas proyecciones epistemológicas apuntan a desafíos actuales, tales como: producir resultados generalizables y acercarse a la Educación Básica; desarrollar repositorios de tendencias de investigación; aumentar la cantidad de investigación de redes y la producción de materiales adecuados para quienes no les gustan las matemáticas.

Palabras clave: Educación Matemática, Enfoques metodológicos, Epistemología de la Educación Matemática.

\section{INTRODUÇÃO}

O desenvolvimento de estudos teóricos e metodológicos para o aperfeiçoamento e desenvolvimento do ensino da matemática é objeto de intensas pesquisas em instituições e grupos colaborativos no mundo inteiro. Esses encaminhamentos podem configurar verdadeiros espaços para uma reflexão ampla sobre a natureza epistemológica do pensamento matemático e suas conexões com as práticas investigativas e aportes educacionais, com base em experimentações diversas.

O entendimento de como a Pesquisa em Educação Matemática está se desenvolvendo implica na necessidade de aprofundar a percepção sobre as abordagens da Pesquisa em Educação Matemática. A importância do aprofundamento do entendimento das abordagens de pesquisa de qualquer área do conhecimento é inquestionável e a Educação Matemática não é exceção. Deste modo, neste trabalho, buscamos apresentar uma discussão sobre a diversidade das abordagens da Pesquisa em Educação Matemática, com base nas informações extraídas de duas pesquisas (SÁ, 2018; MAFRA, 2018) apresentadas no 5o Simpósio Internacional de Educação Matemática SIPEMAT, ocorrido em 2018, na cidade de Belém/PA. 
A preocupação com a Pesquisa em Educação Matemática é relativamente antiga, conforme Carvalho (1994), o qual já afirmava que era “impossível listar aqui [no seu texto de 32 páginas] todas as linhas específicas de pesquisa em Educação Matemática atualmente no Brasil”. De acordo com suas considerações, a área começa a se formar na década de 60, desenvolvendo-se ao final dos 70 e ao longo dos 80 , com a criação dos primeiros programas de pós-graduação e a criação da Sociedade Brasileira de Educação Matemática - SBEM. Nesse sentido, Santos (2011, p. 1), afirma que, "ocorreu o crescimento gradual da produção científico acadêmica, em particular das dissertações e teses, como também a publicação de artigos em revistas especializadas".

De modo amplo, existem duas finalidades principais para a pesquisa em Educação Matemática: uma, de natureza pragmática e profissional, que visa à melhoria da qualidade do ensino-aprendizagem da Matemática nos diversos níveis de ensino; e a outra, de natureza científica, que visa produzir sistematicamente conhecimento rigoroso sobre os diversos aspectos e fatores associados ao ensino-aprendizagem da Matemática. O cumprimento destas finalidades demanda uma série de questionamentos, principalmente, sobre os aspectos teóricos e metodológicos envolvidos nas pesquisas (MATOS, 2001 apud SANTOS, 2011, p. 2).

A pesquisa em educação matemática é multidisciplinar, neste sentido, pesquisadores de diferentes comunidades - psicologia, sociologia, antropologia, matemática, linguística, e epistemologia - contribuem para as construções teóricas desenvolvidas inicialmente fora do campo do ensino e da pesquisa em educação. Como consequência, não é fácil para os pesquisadores em educação matemática delimitar[em] o objeto das suas pesquisas, mesmo que eles próprios o restrinjam no âmbito do ensino e aprendizagem da matemática, depois de considerar a diversidade dos seus determinantes (ARTIGUE, 2005 apud SANTOS, 2011, p.4).

Diferentes abordagens, nesse sentido, permitem estabelecer caracterizações iniciais e inerentes aos processos de mobilização e dinâmica do pensamento matemático, visando o alcance de objetivos e finalidades diversas. Em contraste, é possível verificar que toda a diversidade de abordagens discutidas aqui, trará certamente elementos para se configurar novas perspectivas para o pensar e para a construção do conhecimento dos educadores matemáticos. Assim, este trabalho, também procura apresentar elementos referenciais para se pensar e refletir sobre a atividade de pesquisa educacional, relativa à educação matemática - ancorada em desafios e perspectivadas para a área - como base em um entendimento e a compreensão de amplitudes de diversidades destas pesquisas, observadas em diferentes referenciais (KILPATRICK, 1994; ENGLISH, 2014). 
Esperamos assim, por meio de uma breve revisão destas diferentes abordagens, projetar uma dinâmica de perspectiva para a área, com base em seus desdobramentos epistemológicos e diferentes direcionamentos, o que caracterizaria, de certa forma, uma espécie de espalhamento epistemológico, tal como proposto em Mafra (2018). Tal espalhamento, em nosso entendimento, configura uma diversidade de abordagens de pesquisas, ao detectarmos uma enorme diversidade de propostas, por meio de diferentes amplitudes de procedimentos, técnicas, referenciais e inferências.

\section{UM BREVE HISTÓRICO DA EDUCAÇÃO MATEMÁTICA}

Segundo os registros da memória de Ubiratan D’Ambrósio, registrados em Miguel, Garnica, Igliori e D'Ambrósio (2004), as preocupações com o ensino da matemática foram identificadas na Antiguidade, na idade média, no Renascimento e nos primeiros anos da idade moderna. Sendo que na idade moderna tais preocupações podem ser melhor localizadas.

Na oportunidade, D’Ambrósio ressaltou que após a Revolução Industrial (1767), a Revolução Americana (1776) e a Revolução Francesa (1789) preocupações com a Educação Matemática da juventude começaram a se consolidar, e que a identificação da Educação matemática como área prioritária da Educação ocorreu na transição do século XIX para o século XX. Naquele relato, D’Ambrósio afirmou que são devidos a John Dewey os passos que abrem essa nova área de pesquisa. Destacou ainda que em 1901 em uma reunião da British Associaton, o cientista John Perry disse "ser imensamente importante considerar que a adoção de um método de ensino elementar deve satisfazer um jovem, entre mil, que gosta de raciocínio abstrato, mas que é igualmente importante que os demais não sejam prejudicados.". Durante o relato, Perry foi categórico ao afirmar que "A crise e os conflitos de opinião sobre as reformas na educação estimulam pesquisadores matemáticos de importância, alguns provavelmente preocupados com a educação dos filhos, a se interessarem pelo ensino da matemática" e que, "o passo mais importante no estabelecimento da educação matemática como uma disciplina é devido à contribuição do eminente matemático alemão Felix Klein (1849-1925). Klein, publicou, em 1908, um livro seminal, Matemática elementar de um ponto de vista avançado.". Onde o autor defendia, segundo o entrevistado: (a) uma apresentação (do conhecimento matemático) nas escolas com mais bases psicológicas que sistemáticas; (b) que o professor devia ser um diplomata levando em conta o 
processo psíquico do aluno, para poder agarrar seu interesse e (c) que o professor só teria sucesso ao apresentar as coisas de uma forma intuitivamente compreensível.

Ainda, segundo D’Ambrosio, conforme Miguel et al. (2004), a Educação Matemática como uma subárea da Matemática e da Educação, de natureza interdisciplinar ocorreu com a fundação da Comissão Internacional de Instrução Matemática, conhecida pelas siglas IMUK/ ICMI, durante o Congresso Internacional de Matemáticos, realizado em Roma, em 1908, sob liderança de Felix Klein. Provocando assim a busca por um espaço adequado para Educação Matemática.

No prosseguimento do relato (MIGUEL et al., 2004), D’Ambrósio informou que apesar do crescimento do volume de pesquisa em Educação Matemática até o pós-guerra para pesquisadores em educação matemática, o ambiente era pouco convidativo, tanto nas reuniões anuais do National Council of Teachers of Mathematics (NCTM) quanto nas da American Mathematical Society (AMS) e da Mathematical Association of America (MAA). As reuniões da American Educational Research Association (AERA) ofereciam o ambiente adequado para as pesquisas avançadas, que tomavam grande vulto na época e que somente após a segunda guerra mundial a educação matemática teve efervescência em todo o mundo. Em 1969, realizou-se em Lyons o Primeiro Congresso Internacional de Educação Matemática (ICME1) e desde então, a cada quatro anos, reúne-se um ICME, com a presença de pesquisadores em educação matemática de todo o mundo e organizado sob a responsabilidade da Internacional Commission of Mathematics Instruction (ICMI), uma das comissões especializadas da International Mathematics Union (IMU).

Segundo D’Ambrósio (MIGUEL et al. 2004), no Brasil a Educação Matemática se consolidou por meio da luta vitoriosa dos professores Benedito Antônio da Silva, Maria Cristina de Souza Albuquerque Maranhão, Sandra Pinto Magina, Saddo Ag Almouloud, Silvia Dias Alcântara Machado, Sonia Barbosa Camargo Igliori e Tânia Maria Mendonça Campos, da PUC-SP, que decidiram em 1997, acertadamente, propor à Associação Nacional da Pesquisa em Educação - ANPEd a criação de um GT em Educação Matemática. Iniciativa esta, que tinha como objetivo superar o círculo vicioso que estava formado entre a produção e a divulgação dos resultados das pesquisas brasileira em Educação Matemática. Para maiores detalhes sobre a criação do GT 19 da ANPEd, recomendamos a leitura de Igliori (2003) e para maior conhecimento sobre a história da SBEM, recomendamos a leitura de Pereira (2005).

Com o avanço da pesquisa brasileira em Educação Matemática, campos de investigação começaram a se delinear e a serem registrados em trabalhos científicos. Em Carvalho (1994), encontramos uma lista com 50 linhas de pesquisas registradas pelas instituições consultadas a 
época pelo autor. Uma classificação indicada em Batanero et al. (1992), citado por Fiorentini e Lorenzato (2006), mostra o tamanho da expansão dos campos e linhas de pesquisa, do ponto de vista internacional, ao apresentar um levantamento exaustivo de trabalhos apresentados em congressos internacionais e de centros de pesquisa ativas, em programas de pós-graduação, agrupados em grupos ou tópicos de pesquisas. O foco na resolução de problemas e nos estudos culturais também ganham força, fomentando uma discussão bastante profícua em relação a cognição e a cultura, projetando investigações matemáticas voltadas para a educação matemática, com base em perspectivas sociológicas e antropológicas, ciência da cognição, contribuições de pressupostos do construtivismo e construcionismo, além das tecnologias educacionais emergentes e, mais recentemente, as tecnologias digitais.

Abordagens mais recentes, como a pedagogia de projetos e as investigações em educação matemática tem fornecido pressupostos relevantes, através de estudos recentes, na perspectiva do trabalho em sala de aula. Transformando-as em verdadeiras oficinas de procedimentos e encaminhamentos didáticos, o trabalho envolvendo projetos e investigações, objetivam a produção conceitual e a apreensão de definições e propriedades de muitos conteúdos matemáticos, especialmente aos ligados a geometria e a aritmética.

\section{A EXPANSÃO DO CAMPO DA PESQUISA QUALITATIVA EM EDUCAÇÃO MATEMÁTICA}

A pesquisa em Educação Matemática assim como a pesquisa em Educação tem uma presença da abordagem denominada de qualitativa muito representativa. Isto não é uma crítica. Em Bicudo (2012), temos uma excelente apresentação de argumentos para justificar a prevalência da abordagem qualitativa da Pesquisa em Educação Matemática.

A abordagem qualitativa tem sido adotada por pesquisadores das diversas áreas da Educação Matemática. Essa prevalência da abordagem qualitativa da Pesquisa em Educação Matemática garante a diversidade de abordagens da referida área de pesquisa.

Os pesquisadores de História Matemática, da Educação Matemática e para Educação Matemática tem utilizado muitas das abordagens da História, com as devidas adaptações aos seus objetos e questões de pesquisa. Em Mendes (2012), é encontrada uma excelente análise da pesquisa relacionada com a História da Matemática e da Educação matemática.

Os pesquisadores de Etnomatemática tem adotado muitos dos métodos da Etnografia em suas pesquisas sobre a matemática produzida pelos diversos grupos culturais. Em Syryczyk (2013) 
temos outra apresentação excelente sobre as técnicas de pesquisa utilizadas na Etnomatemática, tais como as de observação sistemática, assistemática e participante. Um outro exemplo de uma pesquisa desenvolvida nessa perspectiva, em um contexto indígena, é o trabalho produzido por Melo e Gonçalves (2017).

Os estudiosos sobre a Formação de Professores de Matemática têm utilizado as técnicas da pesquisa autobiográfica em suas pesquisas. O trabalho de Rosa e Baraldi (2015) é bem esclarecedor sobre o uso da narrativa autobiográfica na pesquisa sobre formação de professores de matemática. Uma outra perspectiva interessante de investigação é apresentada por Oliveira e Schneider (2018), ao discutir as narrativas digitais, no âmbito da formação inicial de professores de matemática e suas implicações no processo de elaboração de práticas alternativas de ensino.

Estudos sobre a Modelagem na Educação Matemática têm adotado a semiótica como ferramenta de análise. Em Klüber e Burak (2014) é encontrado um excelente panorama da pesquisa em modelagem na Educação Matemática brasileira, enquanto que as investigações voltadas para as Tecnologias no Ensino de Matemática, têm adotado técnicas especificas da área da comunicação para suas análises. Uma obra que apresenta uma proposta de fases no uso de tecnologias digitais na Educação Matemática é Borba, Silva e Gadanidis (2014).

A pesquisa de análise de livros didáticos e práticas pedagógicas no ensino de matemática tem adotado abordagens mais próximas da Teoria Antropológica do Didático. Uma referência recente sobre esta abordagem da Pesquisa em Educação Matemática é Bittar (2017), onde é apresentado um desenho metodológico que permite modelar a atividade matemática presente em um livro didático levando em consideração variáveis fundamentais como a instituição e o indivíduo.

Os pesquisadores sobre o Ensino da Matemática têm utilizado muito de técnicas com análises compatíveis com a Engenharia Didática. Nos Encontros Nacionais de Estudantes de Pósgraduação em Educação Matemática - EBRAPEM, ocorridos entre 2014 e 2016, é possível verificar, com base na leitura dos Anais destes eventos, o crescente aumento de pesquisas, sustentadas pelos pressupostos da Engenharia Didática, conforme Sá (2018). Assim como a Engenharia Didática, as pesquisas com foco na Resolução de Problemas, tem possibilitado a compreensão do que seja um problema e suas resoluções, a partir de inúmeras abordagens.

Outras abordagens, não menos importantes, tem oferecido possibilidades de investigação, dentro da educação matemática, que vão desde a pesquisa em fontes históricas até a análise de erros, passando pela metacognição. Sobre a metacognição e suas implicações para a pesquisa em educação matemática, vale a leitura de Darsie (2017). 
Diversas pesquisas têm utilizado a Análise de Erros em suas análises. Uma valorosa referência sobre o tema é Curry (2016) onde são encontrados relatos de pesquisa em Educação matemática que utilizaram a análise de erros. Além disso, as pesquisas em Letramento Matemático tem adotado diversos referencias teóricos, incluindo a Teoria da Objetivação. Sobre o tema, uma referência é Moura, Sforni e Araújo (2011).

Outra teoria que tem crescido bastante nas pesquisas da área de Educação Matemática é a Teoria da Atividade, em particular a Teoria da Formação Planejada das Ações e dos Conceitos de Galperin. Inclusive já contando com um trabalho de Estado da Arte de tal teoria nas pesquisas em Educação Matemática publicado em Gonçalves e Núñez (2017).

Também temos um crescente aumento do número de pesquisas comparativas tendo como exemplo o trabalho de Dias (2016), Dias e Gonçalves (2017), e uma referência mais geral sobre o tema, indicada em Bray, Adamson e Mason (2015).

Assim fica claro que sob o manto da pesquisa qualitativa, temos uma diversidade enorme de abordagens da Pesquisa em Educação Matemática que enriquece e engrandece a produção da área. A oportunidade não permite deixar de registrar que a entrevista, a observação e demais técnicas de pesquisa têm também sido utilizadas em pesquisa da área em questão.

\section{A PESQUISA QUANTITATIVA EM EDUCAÇÃO MATEMÁTICA}

A pesquisa quantitativa em Educação Matemática tem registrado menor ocorrência mais não esteve totalmente ausente. Em Utsumi et al. (2007) temos os resultados de uma pesquisa que apontou fragilidades dos trabalhos de natureza quantitativa nas reuniões anuais da ANPEd no período de 1998 a 2004. As autoras da pesquisa concluíram que em vários dos trabalhos analisados: a) os objetivos e/ou o delineamento não estavam descritos com clareza e b) o referencial teórico encontrava-se isolado na introdução, não sendo utilizado nem para discutir os dados, nem para enfatizar as considerações finais. Além disso, também registraram como fragilidades encontradas dos trabalhos analisados: a) uso inadequado da porcentagem, quando para poucos sujeitos; b) tabelas e/ou gráficos não numerados ou nomeados adequadamente, com erros de conteúdo e/ou formatação, subutilizados na análise; c) notações estatísticas incompletas e/ou com erros -mais diretamente relacionados a análise quantitativa propriamente dita, poderiam ser resolvidos com a leitura dos trabalhos por um parecerista que trabalhasse com esse tipo de análise. 
Estes achados não desqualificaram os trabalhos de natureza quantitativa, mas certamente tornaram mais difícil a aprovação deles no referido evento devido a recomendação de parecerista com trabalho de análise quantitativa para estes tipos de trabalho. Isto poderia ser verificado com uma nova pesquisa de mesma natureza para avaliar os efeitos das conclusões do trabalho de Utsumi et al. (2007) sobre as investigações quantitativas no GT19 da ANPEd após 2007, ano da publicação dos resultados da pesquisa.

Um exemplo de trabalho quantitativo vinculado a Educação Matemática é o de Ortigão (2005), desenvolvido a partir de uma pesquisa do tipo survey (um tipo de método em que se obtém informações diretamente de pessoas, através de diferentes instrumentos de pesquisa, geralmente um questionário) e que objetivou compreender o que professores de matemática selecionam para o ensino dessa disciplina e em que medida esta seleção é impactada por características do perfil socioeconômico e cultural dos estudantes da escola.

Outro exemplo de pesquisa quantitativa em Educação Matemática no Brasil é Andrade, Brandão e Martins (2017) onde são encontrados os resultados de uma pesquisa que usou a regressão logística (trata-se de um técnica, utilizada em estatística, que tem como objetivo, produzir, a partir de um conjunto de observações, um modelo que permita a predição de valores tomados por uma variável categórica) para avaliar as características comuns entre os estudantes com baixo desempenho na Prova Brasil de 2011.

Sobre a pesquisa quantitativa em Educação no Brasil vale analisar o que foi afirmado por Gatti (2004), sobre o tema:

O uso de dados quantitativos na pesquisa educacional no Brasil nunca teve, pois, uma tradição sólida, ou uma utilização mais ampla. Isto dificultou, e dificulta, o uso desses instrumentais analíticos de modo mais consistente, bem como dificulta a construção de uma perspectiva mais fundamentada e crítica sobre o que eles podem ou não podem nos oferecer; dificulta ainda a construção de uma perspectiva consistente face aos limites desses métodos, limites que também existem nas metodologias ditas qualitativas os quais, em geral, não têm sido também considerados. De outro lado, dificulta a leitura crítica e contextuada quando dados quantitativos são trazidos à discussão, seja nos âmbitos acadêmicos, seja em âmbito público. (GATTI, 2004, p.12)

A nossa experiencia nos leva a crer que a situação relatada por Gatti (2004), para a Pesquisa em Educação pode ser transladada sem grandes dificuldades para a Pesquisa em Educação Matemática. Isso talvez explique a predominância das pesquisas de abordagens qualitativas na Pesquisa em Educação Matemática. Entretanto, vale ressaltar que a situação na Pesquisa em 
Educação Matemática tem sofrido mudança no que concerne o uso de técnicas quantitativas de análise de seus resultados.

Bons exemplos desta afirmação são os trabalhos de Araújo e Igliori (2012) e Araújo e Igliori (2013), que apresentam a lógica dos conjuntos difusos como uma alternativa viável de ser agregada à prática da Pesquisa em Educação Matemática, devido a característica da acentuada heterogeneidade do universo da Pesquisa em Educação Matemática.

\section{A PESQUISA MISTA EM EDUCAÇÃO MATEMÁTICA}

A pesquisa ou investigação mista (mixed methods) é outra abordagem que já marca presença na Pesquisa em Educação Matemática. A abordagem mista de pesquisa é uma alternativa de superação da dicotomia entre pesquisa qualitativa e pesquisa quantitativa, devido utilizar simultaneamente técnicas de produção e de análise das informações da pesquisa qualitativa e quantitativa. De modo geral uma referência sobre essa abordagem de pesquisa é Creswell (2007).

Exemplos de pesquisas empregando os métodos mistos, estão indicados em estudos na perspectiva relacional e de compreensão dos aspectos do pensamento e da aprendizagem de discentes e estão no centro de ações metodológicas relacionadas ao que é chamado de research design in mathematics education. Ver, por exemplo, discussões sobre essa perspectiva de abordagem nos Anais do 9th International Mathematics Education and Society Conference (MES9), editado por Chronaki (2017). Neste congresso, realizado em Volos, na Grécia, no ano de 2017, foi possível verificarmos o quanto de discussões desenvolvidas em estudos relacionados entre a educação matemática e a sociedade é pertinente, em muitas pesquisas geradas pelos grupos de estudos, no mundo todo. A cultura, a política e a ética apresentam subsídios cada vez mais profundos em se tratando de estudos voltados para o ensino e a aprendizagem da matemática.

Outro exemplo, de mixed methods, aparece em estudos cada vez mais presentes como, por exemplo, o learner's perspective study (exame de interações e participação em sala de aula, a partir de determinadas situações problemas propostos), em uma perspectiva de ambientes educativos inovadores, tal como o proposto em Pedro (2017). Em adição, estudos com base metodológica investigativa, fundamentada nos métodos mistos, são os estudos comparativos em matemática, pesquisas sobre acessibilidade e matemática, tecnologias digitais relacionadas a educação matemática, tais como os aparelhos móveis, robótica, distance learning (ensino à distância) e e- 
learning (trata-se de um modelo de ensino não presencial, apoiados em um rol de tecnologias diversas).

Além destas, pesquisas envolvendo blended learning - cujo o foco está relacionado com um sistema de formação onde parte dos conteúdos é transmitido em cursos à distância, normalmente pela Internet, em que são incluídas sessões presenciais - estudos voltados para a psicologia e a filosofia da educação matemática, bem como os estudos culturais, assim como a etnomatemática, apresentam desdobramentos diversos, voltados para o entendimento e a compreensão de práticas socioculturais identificadas e localizadas em diferentes regiões do planeta. Todas essas abordagens e exemplos podem ser conhecidos, com base na leitura dos Topic Study Groups (TSG), organizados em ICME-13 (KAISER, 2017).

Outras abordagens com base nos métodos mistos, tratam da utilização de experimentos de ensino, conforme Steffe \& Tomphsom (2000), além de técnicas de análises de fitas de vídeo, pesquisa documental, elementos da pesquisa-ação, história oral e observações etnográficas. Além disso, ganham corpo o estudo da educação matemática, no nível da educação infantil - através de investigações configuradas por meio de estudos de caso e o uso e manipulação de tecnologias digitais - visando uma compreensão de representações espaciais, em crianças.

Os trabalhos de Oliveira (2012) e Pelli (2014), são exemplos de pesquisas de abordagem mista em Educação Matemática realizadas no Brasil e, Rosa (2010) é um exemplo de pesquisa mista em Educação Matemática realizada por brasileiro fora do Brasil.

Por fim, investigações envolvendo questões intrínsecas ao contexto escolar, tais como os estudos de gênero, saberes informais, gamificação e tecnologias digitais, tais como o de Sousa, Viali e Ramos (2017), ganham força e se mostram cada vez mais presente no âmbito da epistemologia do conhecimento, mais especificamente para a área da Educação e da Educação Matemática.

Assim, o campo da pesquisa mista, em educação matemática, apresenta elementos multi referenciados e difíceis de serem prognosticados. Além disso, entendemos que a abordagem mista, aproxima-se de uma perspectiva holística, nos moldes do estudo de Rudd e Goodson (2016), fornecendo assim aspectos de análises e inferências em explorações metodológicas e teóricas, as quais entendemos serem cada vez mais complexas. 


\section{DESAFIOS DA PESQUISA EM EDUCAÇÃO MATEMÁTICA}

Como toda área do conhecimento em plena atividade a Educação Matemática tem os seus desafios específicos em cada momento histórico. No início, a Educação matemática esteve muito ligada as questões diretamente associadas a busca do aperfeiçoamento do processo de ensino, aprendizagem e avaliação da matemática escolar. Depois da solidificação da área de pesquisa e a ampliação das abordagens, conforme mostramos anteriormente, essa ligação ficou um pouco menos estreita. $\mathrm{O}$ que não é um demérito.

Em 1993, para Cunha (1993 apud Carvalho 1994), era "a escola normal o elemento mais importante para uma ação política educacional visando à melhoria do ensino da Matemática".

Com base em nossa vivência na Educação Matemática brasileira, apresentaremos os desafios que consideramos ainda atuais à Pesquisa em Educação Matemática.

Os desafios para a Pesquisa em Educação Matemática visualizados por nós são os seguintes:

(a) produzir resultados generalizáveis

A pesquisa em Educação Matemática por ter sido predominantemente qualitativa traz consigo o custo de que muitos resultados obtidos não possam ser generalizados. Isto faz com que excelentes produtos não sejam incorporados ao fazer pedagógico do professor de matemática. Este desafio implica na necessidade de pesquisa de replicação simultânea de estudos visando produzir informações para grupos maiores visando possibilitar o uso de testes estatísticos dos resultados obtidos. Os resultados bem-sucedidos poderão justificar inclusive a aquisição do material produzido, testado e avaliado por órgãos do governo tal como é realizado com o livro didático.

(b) produzir resultados que estreitem os laços com a Educação Básica

Este desafio está ligado a necessidade de ouvirmos mais os nossos colegas docentes da Educação Básica sobre os problemas e dificuldades convividos pelos mesmos e realizar pesquisa a partir dessa escuta. Certamente surgirão problemas de pesquisa de extrema relevância que nunca foram imaginados nos meios acadêmicos.

(c) desenvolver repositórios das tendências de pesquisa

Assim como a modelagem matemática e a história da matemática já fizeram é importante que as outras tendências consolidadas da Educação Matemática construam seus repositórios. Isto ampliará as possiblidades de realização de pesquisa em cada tendência devido a disponibilidade de materiais para pesquisa. 
(d) aumentar a quantidade das pesquisas em rede

O aumento da quantidade de redes de pesquisa em Educação Matemática é uma necessidade de todas as regiões do nosso país, principalmente da região Amazônica. A ampliação de redes de pesquisa vai aumentar a capacidade de captação de recursos, publicação conjunta, análises de situações regionais e fortalecer a área.

(e) produzir resultados na direção da criação de protocolos profissionais

Um dos fatores que prejudicam muito a atividade dos docentes é a falta de protocolos estabelecidos a partir da pesquisa como acontece em outras profissões. Isto fragiliza a área devido deixar espaço para práticas já comprovadas como ineficazes e prejudiciais aos estudantes. Por este motivo precisamos desenvolver estudos que fortaleçam a organização profissional para 0 fortalecimento da mesma.

(f) Associar amiúde a pesquisa com a extensão

A divulgação dos resultados de pesquisa por meio de trabalhos em periódicos, anais de eventos é de grande importância e merece todo o esforço da comunidade de pesquisadores da área. Entretanto, é necessário que as conclusões, sugestões, materiais e abordagens frutos das pesquisas sejam socializados com a comunidade interessada com maior frequência e no formato de divulgação. Assim os interessados que estão distantes do ambiente de pesquisa terão oportunidade de conhecer, de forma menos acadêmica, orientações, materiais e resultados que poderão ser incorporados ao cotidiano das suas atividades como docentes e atrair também novos interessados na pesquisa.

(g) Não esquecer das origens

Em alguns momentos temos a impressão que a Pesquisa em educação matemática está indo em direção somente de aprofundar seu repertório. Isto é perigoso. Devemos sempre lembrar da origem da Educação Matemática com seu forte compromisso social. Ver, por exemplo, o trabalho de Silva e Godoy (2016). Do contrário perderemos muitos dos interessados em Educação Matemática para outros movimentos, com discurso de preocupação com os problemas da Educação Básica. Vale ressaltar que essa expectativa de olhar com atenção para Educação Básica não é um privilégio da área da Educação Matemática. Na área de Ensino de Ciências a expectativa ainda é a mesma. O registro dessa expectativa ocorreu e foi reiterado recentemente em Anjos, Pereira e Roças (2018).

(h) Evitar atritos entre abordagens de pesquisa 
Devido a Educação Matemática ser multidisciplinar ela abriga, como devia ser, práticas e concepções de escolas das mais variadas formas, entre elas a didática francesa, a influência americana, entre outras. Temos percebido em alguns momentos ações e discursos que provocam ressentimentos entre pesquisadores que adotam posturas de origens diferentes. Esta ocorrência não ajuda o avanço da área e nem fortalece o entendimento das vantagens e limitações de cada corrente de pesquisa. Tudo que não precisamos é de divisão. Precisamos sim estarmos abertos ao entendimento das críticas e sugestões oriundas de pesquisadores que tem ponderações a apresentar. Essas ponderações devem ser apresentadas e debatidas nos encontros de pesquisa com intuito aprofundar o entendimento dos interessados sobre os conceitos fundantes de cada corrente de pensamento na busca de aprimorar a nossa prática de pesquisa.

(i) Produzir materiais adequados para quem não gosta de matemática

A nossa experiencia em Educação Matemática tem mostrado que temos uma dívida histórica com as pessoas que dizem não gostar de matemática ou apresentam dificuldades com as ideias, métodos e processos da mesma. Muitas ações nesta direção já foram desenvolvidas, mas ainda não conseguimos elaborar materiais, livros, textos, vídeos, que atendem as necessidades das pessoas que não tiveram a oportunidade de desenvolver apreço ou segurança no enfrentamento de situações que envolvam o conhecimento matemático mesmo que elementar. Essa dívida quando for paga trará para a Educação Matemática como um todo, resultados muito gratificantes, uma vez que se conseguirmos diminuir o sentimento de insegurança com a matemática de nossa população teremos dado uma grande contribuição para o alcance de sua autonomia técnico-política. Uma boa discussão, nessa direção, encontramos em Souza (2016).

(j) Praticar mais a pesquisa de desenvolvimento

A pesquisa de desenvolvimento traz a oportunidade de uma resposta às críticas de que a pesquisa educacional não tem contribuído muito para enfrentar efetivamente os problemas educacionais, em particular do processo de ensino, aprendizagem e avaliação de conteúdos de matemática da escola básica. Como a pesquisa de desenvolvimento permite acontecer em etapas que resultam em aperfeiçoamento de produtos educacionais desenvolvidos como consequência do acumulo entre testagem, revisão teórica e refinamento do produto desenvolvido ela permite o desenvolvimento de estudos mais amplos com participação de docentes da Educação Básica que poderão avaliar o material elaborado, sua viabilidade de utilização, sua funcionalidade e os efeitos educacionais obtidos em parceria com os pesquisadores responsáveis pelo desenvolvimento do 
produto. Uma das consequências de superarmos este desafio será uma maior aproximação entre a educação básica e a pesquisa em Educação Matemática.

(k) Aperfeiçoamento metodológico e maturidade do pesquisador em educação matemática

Uma questão que parece bastante pertinente, é pensar sobre quais tipos de métodos e procedimentos apresentam bastante frequência na pesquisa educacional. Mais especificamente, em educação matemática, quais métodos são mais apropriados para abordar questões $A, B, C$ e não questões $X, Y, Z$. Tais elementos espera-se estar diretamente conectados com os referenciais adotados nos estudos os quais utilizaram tais métodos, com o uso racional e habilidade esperada, na condução e delineamento das investigações. Isso implica em termos profundidade e foco de investigação visando as discussões relacionais a produção do conhecimento. Métodos de pesquisa são melhor escolhidos e adaptados as nossas necessidades, quando se tem uma ideia do que se está procurando ou delineando, visando a produção de conhecimento. De acordo com Schoenfeld (2008):

Um método de pesquisa é uma lente através da qual alguns conjuntos de fenômenos são vistos. Uma lente pode trazer alguns fenômenos nitidamente em foco. Mas também pode borrar outros ao mesmo tempo, e talvez até criar imagens artificiais ou ilusórias (SCHOENFELD, 2008, p. 476, tradução nossa).

Nesse sentido, métodos estão correlacionados com teorias. Independente se é utilizado, em um estudo a abordagem $\alpha, b$ ou $\mu$ na qual se utilizam técnicas e instrumentos $\pi, \rho$ ou $\sigma$. Um aspecto crucial em se tratando de validação e originalidade de uma pesquisa é verificar se técnicas e instrumentos fazem sentido e se há diálogo entre os instrumentos metodológicos, de tal forma que a configuração metodológica (que pode variar de pesquisa a pesquisa) permita estabelecer organicidade e parâmetros satisfatórios de confiabilidade e de respostas aos objetivos propostos.

\section{CONSIDERAÇÕES FINAIS MOMENTÂNEAS}

As considerações que apresentaremos neste momento estão longe de ser finais. Dado que a temática abordada é caracterizada pela impermanência de todas os movimentos e coisas da vida. A elaboração deste texto foi de grande valia pessoal por nos ter obrigado a atentar para aspectos de nossa prática que no cotidiano têm passado despercebidos, de modo que consideramos este desafio como uma oportunidade genuína de aprendizagens e reflexões. 
O momento atual da pesquisa em Educação Matemática no Brasil é fruto das inquietações e da persistência de seus atores e as pesquisa em educação matemática certamente trilharão os caminhos que forem possíveis, dentro das condições materiais e históricas na qual o momento presente permita construir e direcionar.

A certeza que temos é que, a Pesquisa em Educação Matemática no Brasil continuará a sua caminhada sempre marcada pelo compromisso de aperfeiçoamento constante, apesar de todos os entraves de cada momento como tem sido mostrado pela trajetória desta área de pesquisa em nosso país, sempre tendo a diversidade de abordagens da pesquisa como uma das características positivas para o fortalecimento, crescimento e respeitabilidade da referida área.

As abordagens, concepções e perspectivas apresentadas nesse trabalho não se encontram isoladas. Essas diretrizes indicam, de acordo com os estudos e pesquisas consultadas, que algumas se inter-relacionam e podem (na verdade, devem) ser desenvolvidas de uma forma efetivamente bricolada em diferentes ambientes de aprendizagens e em grupos colaborativos de pesquisa voltados para o ensino a aprendizagem de matemática.

Embora os estudos em educação matemática nos permitam discutir e revisitar o ambiente escolar sob múltiplas perspectivas e diversidades de olhares, é extremamente difícil, traçarmos um panorama conclusivo sob o futuro da educação matemática no Brasil e no Mundo.

Entendemos que muitos problemas, que encontramos no ensino e aprendizagem da matemática por parte de alunos e professores não estão diretamente relacionados com a matemática em si, mas sim, decorre de vários aspectos adjacentes e periféricos, existentes na conjuntura envolvida em um espaço matemático de aprendizado conceitual. Esses problemas, acreditamos, serão gradativamente reduzidos, a medida em que estivermos dispostos a entender e a compreender, a cada momento, de que:

É necessário discutir e refletir sobre nossas experiências locais com o aprendizado da matemática, envolvendo diferentes perspectivas de trabalho;

Agregar essas experiências ao aperfeiçoamento continuo de práticas docentes, visando a incorporação ou o surgimento de novos procedimentos metodológicos;

$>\quad$ Observar que os problemas com o ensino da matemática não podem requerer uma discussão isolada, pois observa-se um nível de tensão e correlação muito forte entre essa área de conhecimento e outras.

Além disso, espera-se que a amplitude de pesquisas e abordagens aqui apresentadas, possa fornecer discussões e analises visando facilitar aos discentes, por meio de práticas de ensino 
docentes, uma compreensão maior dos aspectos intrínsecos aos processos educacionais. Naturalmente o caminho não é fácil más, os desafios se tornam importantes para o processo de amadurecimento e consistência de atributos essenciais e bagagem de conhecimento aos pesquisadores em educação matemática.

Assim, consideramos evidente um espalhamento epistemológico, em termos das pesquisas e estudos em educação matemática, os quais estão refletidos em diferentes abordagens e epistemologias. Considerações nesse sentido, fornecem uma dimensão do quanto os movimentos são diversos, projetam experiências susceptíveis de se tornarem uma fonte de informação, esclarecimentos e inspiração para as pessoas envolvidas e compromissadas com o futuro da educação matemática no Brasil e no Mundo.

\section{REFERÊNCIAS}

ANDRADE, Doherty; BRANDÃO, Bernadete Maria Suaki e MARTINS, Ana Beatriz Tozzo. Características comuns entre alunos com baixo rendimento em matemática na Prova Brasil de 2011: um estudo logístico. Revista Paranaense de Educação Matemática, v.6, n.10, p.204-222, jan-jun 2017.

ANJOS, Maylta Brandão dos; PEREIRA, Marcus Vinicius e RÔÇAS, Giselle. Nós que aqui estamos por vós esperamos: a desejada aproximação entre educação básica e pesquisadores em ensino de ciências. Espaço Pedagógico. v.25, n.2, Passo Fundo, p.528-545, maio/ago. 2018. Disponível em www.upf.br/seer/index.php/rep.

ARAÚJO, Péricles César de e IGLIORI, Sonia Barbosa Camargo. Complementaridade: pesquisa qualitativa e quantitativa na educação matemática. Revista de Produção Discente em Educação Matemática., São Paulo, v.1, n.1,p.113-122, 2012.

ARAÚJO, Péricles César de e IGLIORI, Sonia Barbosa Camargo. Métodos de pesquisa combinados em Educação Matemática. In: XI Encontro Nacional de Educação Matemática. Anais... Curitiba 2013.

BICUDO, Maria Aparecida Viggiani. A pesquisa em educação matemática: a prevalência da abordagem qualitativa. Revista Brasileira de Ensino de Ciência e Tecnologia, vol 5, n.2, p.16-26, mai-ago. Caxambu. 2012.

BITTAR, Marilena. A Teoria Antropológica do Didático como ferramenta metodológica para análise de livros didáticos. ZETETIKÉ (ON LINE), v. 25,n.3, p. 363-387, 2017.

BORBA, Marcelo de Carvalho; SILVA, Ricardo Scucuglia Rodrigues da e GADANIDIS, George. Fases das tecnologias digitais em Educação Matemática: sala de aula e internet em movimento. BH: Autentica, 2014, 149p.

BRAY Mark; ADAMSON Bob; MASON Mark (Orgs.). Pesquisa em Educação Comparada: abordagens 
e métodos. Brasília: Líber Livro, 2015.

CARVALHO, João Pitombeira de Avaliação e Perspectivas da área de Ensino de Matemática no Brasil. Em Aberto. Ano 14, n. 62, p.74-88, abr./jun. 1994.

CHRONAKI Anna. (Ed.) Proceedings of the ninth international mathematics education and society conference. MES9. Proceedings... Volos, Greece: University of Thessaly Press, 2017.

CRESWELL, John Projeto de pesquisa: métodos qualitativo, quantitativo e misto. Tradução de Luciana de Oliveira da Rocha. 2 ed. Porto Alegre: Artmed, 2007.

CURRY, Helena Noronha. Erros na aprendizagem de matemática: relatos de pesquisas e reflexões. 1. ed. Santa Maria: Centro Universitário Franciscano, 2016. v. 1. 194p.

DARSIE, Marta Maria Pontin. A metacognição como estratégia de superação de crenças e sentimentos negativos em relação à matemática: a aprendizagem matemática no curso de pedagogia. LATIN AMERICAN JOURNAL OF SCIENCE EDUCATION, v.4, p.1-13,2017.

DIAS, Ana Lúcia Braz e GONÇALVES, Harryson Júnio Lessa. Contribuições da Educação Comparada para Investigações em Currículos de Matemática. Educação Matemática Pesquisa., São Paulo, v.19, n.3, p.230-254, 2017.

DIAS, Marcelo. Tendências em Educação Matemática: percursos curriculares brasileiros e paraguaios. Curitiba: Appris, 2016, 245p.

ENGLISH L. (Ed.) Handbook of international research in mathematics education. Nova York: Ed. Routledge Taylor \& Francis Group, 2014.

FIORENTINI, Dario e LORENZATO, Sérgio. Investigação em educação matemática: percursos teóricos e metodológicos. Campinas/SP: Autores Associados, 2006. (Coleção formação de professores)

GATTI, Bernadete Angelina. Estudos quantitativos em Educação. Educação e Pesquisa. São Paulo, v.30, n.1, p.11-30, jan/abr. 2004.

GONÇALVES, Paulo Gonçalo Farias e NUNEZ, Isauro Beltran. A Teoria de P. Ya. Galperin nas pesquisas em educação matemática. Educação Matemática Debate, Montes Claros, v. 1, n. 3, p. 277-295, set./dez. 2017.

IGLIORI, Sônia Barbosa Camargo. A Criação do Grupo de Trabalho de Educação Matemática na ANPEd: O GT19. In: 26a RA da ANPEd. Anais... Caxambu 2004.

KAISER Gabriele. (Ed.) Proceedings of the 13th International Congress on Mathematical Education, ICME-13. Proceedings... Switzerland: Springer, 2017.

KILPATRICK Jeremy. Investigación em educación matemática: su história y alguns temas de actualidad. In: KILPATRICK, J. RICO L. GÓMEZ, P. (Eds.) Educacion Matemática. México: Grupo Editorial Iberoamérica, 1994. 
KLÜBER, Tiago Emanuel e BURAK, Dionisio. Sobre a pesquisa em Modelagem na Educação Matemática Brasileira. Revista Diálogo Educacional., Curitiba, v. 14, n. 41, p. 143-163, jan./abr. 2014.

MAFRA, José Ricardo. Diversidade de abordagens na pesquisa em educação matemática. In: 5o Simpósio Internacional de Pesquisa em Educação Matemática. Anais... Belém/PA. 2018.

MELO, Elisângela Aparecida; GONÇALVES, Tadeu Oliver. Práticas socioculturais Xerente em comunidades de prática. Revista Exitus, v. 7, n. 2, p. 191-215, mai/ago 2017. http://www.ufopa.edu.br/portaldeperiodicos/index.php/revistaexitus/article/view/311 Acesso em 30 de abr. de 2020

MENDES, Iran Abreu. Pesquisas em história da Educação Matemática no Brasil em três dimensões. Quipu, vol. 14, núm. 1 enero-abril de 2012, pp. 69-92.

MIGUEL, Antonio; GARNICA, Antonio Vicente Marafioti; IGLIORI, Sonia Barbosa Camargo e D'AMBRÓSIO, Ubiratan. A educação matemática: breve histórico, ações implementadas e questões sobre sua disciplinarização. Revista Brasileira de Educação. Set /Out /Nov /Dez 2004 n. 27 p:70-93.

MOURA, Manoel Oriosvaldo de, SFORNI, Marta Sueli de Faria e ARAÚJO, Elaine Sampaio. Objetivação e apropriação de conhecimentos na atividade orientadora de ensino. Revista Teoria e Prática da Educação, v. 14, n. 1, p. 39-50, jan./abr. 2011.

OLIVEIRA, Carloney e SCHNEIDER, Henrique. As Narrativas Digitais com o apoio de Infográficos na formação inicial do pedagogo nas aulas de Matemática: Narrar, Informar e Compartilhar. Revista Tempos e Espaços em Educação. São Cristóvão, Sergipe, Brasil, v. 11, n. 01, Edição Especial, p. 169186, dezembro, 2018. http://dx.doi.org/10.20952/revtee.v11i01.9586 Acesso em 30 de abr. de 2020.

OLIVEIRA, Davidson Paulo Azevedo. Um estudo misto para entender as contribuições de atividades baseadas nos fundos de conhecimento e ancoradas na perspectiva sociocultural da história da matemática para a aprendizagem de funções por meio da pedagogia culturalmente relevante. Dissertação (Mestrado Profissional em Educação Matemática). Ouro Preto, MG: UFOP, 2012. 311p.

ORTIGÃO, Maria Isabel Ramalho. Currículo de matemática e desigualdades educacionais. Tese (Doutorado em Educação) - Departamento de Educação, Pontifícia Universidade Católica do Rio de Janeiro. 2005. $194 \mathrm{f}$.

PEDRO, Neuza. Ambientes educativos inovadores: o estudo do fator espaço nas 'salas de aula do futuro' portuguesas. Revista Tempos e Espaços em Educação, São Cristóvão, Sergipe, Brasil, v. 10, n. 23, p. 99-108, set./dez. 2017. http://dx.doi.org/10.20952/revtee.v10i23.7448 Acesso em 11 de mar. de 2020.

PELLI, Débora. As contribuições do software GeoGebra como um medidor do processo de aprendizagem da geometria plana na educação a distância (EAD) em um curso de licenciatura em 
pedagogia. Dissertação (Mestrado Profissional em Educação Matemática). Ouro Preto, MG: UFOP, 2014. 249p.

PEREIRA, Denizalde Jesiél Rodrigues. A História do movimento democrático que criou a Sociedade Brasileira de Educação Matemática (SBEM). Tese (Doutorado em Educação). Campinas: UNICAMP, 2005.

ROSA, Milton. The perceptions of high school leaders about English language learners (ELL): the case of mathematics. 615p. Tese (Doutorado em Educação). Liderança Educacional. Sacramento, CA: CSUS, 2010.

ROSA, Fernanda Malinosky C. da e BARALDI, Ivete Maria. O uso de narrativas (auto)biográficas como uma possibilidade de pesquisa da prática de professores acerca da Educação (Matemática) Inclusiva. Bolema, Rio Claro (SP), v. 29, n. 53, p. 936-954, dez. 2015.

RUDD, Tim; GOODSON, Ivor. Refraction as a Tool for Understanding Action and Educational Orthodoxy and Transgression. Revista Tempos e Espaços em Educação, São Cristóvão, Sergipe, Brasil, v. 9, n. 18, p. 99-110, jan./abr. 2016. http://doi:10.20952/revtee.2016vl9iss17 Acesso em 16 de mar. de 2020.

SÁ, Pedro Franco de. A DIVERSIDADE DE ABORDAGENS DA PESQUISA EM EDUCAÇÃO MATEMÁTICA: aspectos qualitativos, quantitativos e desafios. In: 50 Simpósio Internacional de Pesquisa em Educação Matemática. Anais... Belém/PA. 2018.

SANTOS, Marluce Alves dos.. Discutindo sobre metodologia da pesquisa em educação matemática. XIII CIAEM-IACME Anais..., Recife, Brasil, 2011.

SCHOENFELD Alan. Research methods in (mathematics) education. In: ENGLISH L. (Ed.). Handbook of International Research in Mathematics Education. 2. Ed. Nova York: Ed. Routledge Taylor \& Francis Group, 2008.

SILVA, Cecy Leite Alves da e GODOY, Elenilton Vieira. Tendências De Pesquisa Em Educação Matemática que Privilegiam As Dimensões Social, Cultural E Política Da Matemática Escolar. REnCiMa, Edição Especial: Educação Matemática, v.7, n.4, p. 128-148, 2016.

SOUSA, Emerson Silva; VIALI, Lorí; RAMOS, Maurivan. Construção e análise de modelos exponenciais de forma significativa: uma experiência de ensino em sala de aula. Revista Exitus, v. 7, n. $2, \quad$ p. $55-73, \quad 2017$. http://www.ufopa.edu.br/portaldeperiodicos/index.php/revistaexitus/article/view/302 Acesso em 30 de abr. de 2020

SOUZA, Carla Alves de. Circulação e apropriação de ideias em Educação Matemática: aproximações. Tese (Doutorado em Educação) USP, 2016. 421p.

STEFFE, Leslie e THOMPSON, Patrick, Teaching experiment methodology: Underlying principles and essential elements. In: KELLEY Anthony E. \& LESH Richard. (Eds.) Handbook of research design in mathematics and science education, Mahwah, NJ: Erlbaum 2000. 
SYRYCZYK, Edilberto. Pesquisa Etnomatemática: métodos e técnicas para uma construção socioetnoculturalista. Revista REAMEC, Cuiabá - MT, n.01, p. 73-87 setembro 2013.

UTSUMI, Miriam; CAZORLA, Irene; VENDRAMINI, Claudette e MENDES, Clayde. Questões metodológicas dos trabalhos de abordagem quantitativa apresentados no GT19-ANPEd. Educação Matemática Pesquisa, São Paulo, v. 9, n. 1, p.83-101, 2007.

\section{$\underline{\text { SOBRE OS AUTORES }}$}

\section{José Ricardo e Souza Mafra}

Doutorado em Educação, pela Universidade Federal do Rio Grande do Norte (UFRN). Professor da Universidade Federal do Oeste do Pará (UFOPA), Brasil. Professor permanente do Programa de Pós-graduação em Educação - PPGE/UFOPA e do Programa de Pós-graduação em Educação na Amazônia - Doutorado em Rede - PGEDA/UFPA. Também é docente permanente do Programa de Doutorado em Educação em Ciências e Matemática (PPGECEM) - Associação em Rede - intitulada Rede Amazônica de Educação em Ciências e Matemática (REAMEC). Líder do Grupo de estudos e pesquisas em educação matemática e interdisciplinaridade na Amazônia.

E-mail: jose.mafra@ufopa.edu.br

Orcid: https://orcid.org/0000-0002-3629-8959

\section{Pedro Franco de Sá}

Doutorado em Educação, pela Universidade Federal do Rio Grande do Norte (UFRN). Professor titular da Universidade do Estado do Pará (UEPA), Brasil. Professor permanente do Programa de Pós-graduação em Educação - PPGED/UEPA e do Programa de Pós-graduação (Mestrado Profissional) em Ensino de Matemática - PMPEM/UEPA. Também é docente permanente do Programa de Doutorado em Educação em Ciências e Matemática (PPGECEM) - Associação em Rede - intitulada Rede Amazônica de Educação em Ciências e Matemática (REAMEC). Líder do Grupo de Estudos em Cognição e Educação Matemática.

E-mail: pedro.franco.sa@gmail.com

Orcid: https://orcid.org/0000-0002-8986-2787 